\title{
Estimation of spinodal pressure by extrapolation of Modified general Lennard-Jones equation of state (MGL-J EOS)
}

A. M. AL-Sheikh

H. B. Mohammed

Department of Physics / College of Science

University of Mosul

Received

05 / 01 / 2011
Accepted

06 / 04 / 2011

\begin{abstract}
الخلاصة
تم في هذا البحث وضع صيغة جبرية لاحتساب ضغط السبينودل باستخدام الاستكمال

الرياضي لنتائج تغير معامل المرونة الحجمي مع الضغط والتي تم الحصول عليها باستخدام معادلة الحالة العامة لينارد- جونس المحورة.
\end{abstract}

\section{Abstract}

An expression for spinodal pressure has been formulated by extrapolating variation of bulk modulus with pressure which were evaluated by using modified general Lennard-Jones equation of state (MGL-J EOS).

\section{INTRODUCTION:}

The equation of state (EOS) can be extrapolated into the pressure range in which the material is metastable. This extrapolation gives an estimate of high pressure results which couldn't be obtained experimentally. Moreover, such extrapolation can gives an estimation of the spinodal pressure (i.e.) The pressure at which the bulk modulus go to zero (Maris, 2009).

MGL-J EOS can describe fluid state and is one of important tools to calculate the (PVT) relation and vapor-liquid equilibrium (Yutaka et al., 2007) and have all the merits of ideal universal (EOS) as it is energy analytic, pressure and volume analytic, beside it satisfies the following spinodal condition (Jiuxun, 2005):

$B \propto\left(P-P_{s p}\right)^{1 / 2} \quad$ with $\quad B\left(P=P_{s p}\right)=0$ 
And it is based on the generalized Lennard-Jones (GL-J) potential which is a mathematically simple model that describes the interaction between a pair of neutral atoms or molecules. The most common expression of the GL-J potential is (Lennard-Jones, 1924):

$$
\begin{aligned}
V & =4 \varepsilon\left\{(\sigma / r)^{12}-(\sigma / r)^{6}\right\} \\
& =\varepsilon\left\{\left(r_{m} / r\right)^{12}-2\left(r_{m} / r\right)^{6}\right\}
\end{aligned}
$$

Where:

$(\varepsilon)$ depth of potential well.

$(\sigma)$ distance at which the inter particle potential is zero.

$(r)$ distance between the particles.

$\left(r_{m}\right)$ distance at which the potential reaches its minimum, at $r_{m}$ the potential function has the value $(-\varepsilon)$.

The distances are related as $r_{m}=\left(2^{1 / 6} \sigma\right)$ these parameters can be fitted to reproduce experimental data or accurate quantum chemistry calculations. The $\left(r^{-12}\right)$ term describe the Pauli repulsion at short ranges due to overlapping electron orbitals and the $\left(r^{-6}\right)$ term describes attraction at long ranges (Vander Waals force or dispersion force).

(Jiuxun, 2005) adopt an all neighbor model to replace the nearest neighbor to obtain a modified lennard-jones equation of state (MGL-J EOS) in the form:

$$
P=B o / n(V o / V)^{n}\left[(V o / V)^{n}-1\right]
$$

Where $n=1 / 3 B o^{\prime}$

$B o, B o^{\prime}$ are the bulk modulus and its first derivative at zero pressure

$V o$ is the volume at zero applied pressure; $V$ is the volume at pressure $(P)$.

Equation (3) is energy analytic; pressure and volume analytic, beside it satisfy the spinodal condition of equation (1).

\section{THEORITICAL DETAILS AND RESULTS:}

\section{VARIATION OF BULK MODULUS WITH PRESSURE:}

The bulk modulus of material determines how much it will compress under a given amount of external pressure:

$$
B=-V d p / d V
$$

or means the reciprocal of compressibility. Thus, it is a measure of resistance to compressibility. (Gaudoin and Foulkes, 2002).

From equation (3):

$$
\begin{aligned}
& P=\frac{B_{o}}{n} V_{o}^{n} V^{-n}\left[V_{o}^{n} V^{-n}-1\right]=\frac{B_{o}}{n}\left[V_{o}^{2 n} V^{-2 n}-V_{o}^{n} V^{-n}\right] \\
& \begin{aligned}
d P / d V & =B o / n\left[V o^{2 n}(-2 n) V^{-2 n-1}-V o^{n}(-n) V^{-n-1}\right] \\
& =B o / n\left[-2 n V o^{2 n} V^{-2 n} \cdot V^{-1}+n V o^{n} V^{-n} \cdot V^{-1}\right] \\
& =B o\left[-2(V o / V)^{2 n} 1 / V+(V o / V)^{n} 1 / V\right]
\end{aligned}
\end{aligned}
$$




$$
\begin{aligned}
& =B o / V(V o / V)^{n}\left[-2(V o / V)^{n}+1\right] \\
& =-B o / V(V o / V)^{n}\left[2(V o / V)^{n}-1\right]
\end{aligned}
$$

From equation (5) and (6)

$$
\begin{aligned}
B_{T} & =-V(d p / d V)_{T} \\
& =B o(V o / V)^{n}\left[2(V o / V)^{n}-1\right]
\end{aligned}
$$

\section{EVALUATION OF SPINODAL pressure:}

By relating equation(3) and equation(7) and using values of $B o$ and $B o^{\prime}$ shown in table(1) with (n)evaluated from equation(4) we obtain variation of $B_{T}$ with $V o / V$ and consequently with pressure as shown in figure(1) and figure(2) for $\mathrm{C}$ and $\alpha$-Sn respectively.

Table (1): Experimental values of $B o$ and $B o^{\prime}$ (Tripathi et al., 2006)

\begin{tabular}{|c|c|c|}
\hline material & Bo $\mathbf{( G P a )}$ & $B o^{\prime}$ \\
\hline $\mathrm{C}$ & 442.0 & 4.69 \\
\hline$\alpha-\mathrm{Sn}$ & 53.0 & 4.22 \\
\hline
\end{tabular}

Then by definition of spinodal pressure, as the pressure at which the bulk modulus go to zero, and extrapolating results shown in figure (1) and figure (2), we obtain an estimation for spinodal pressure in the form: $P_{s p}=-\operatorname{BodP} / d B$

Which differ from the estimation given by (jiuxun, 2005):

$P_{s p}=-B o / 4 n$, where $n=1 / 3$, then

$P_{s p}=-0.75 B o / B o^{\prime}$

Figure (3) and (4) shows the values of $P_{s p}$ for $\mathrm{C}$ and $\alpha-\mathrm{Sn}$, respectively, comparison between these values and values obtained by using equation (9) [jiuxun, 2005] shown in table (2):

Table (2): A comparison for values of $P_{s p}$ obtained in present work and other reference.

\begin{tabular}{|c|c|c|}
\hline \multirow{2}{*}{ Element } & \multicolumn{2}{|c|}{$P_{s p}(\mathrm{GPa})$} \\
\cline { 2 - 3 } & Present work & Jiuxun,2005 \\
\hline $\mathrm{C}$ & -113.55 & -70.6 \\
\hline$\alpha-\mathrm{Sn}$ & -15.20 & -9.4 \\
\hline
\end{tabular}

\section{DISCUSSION:}

The practical value of spinodal condition and the importance of volume analyticity can be pointed out as, in the recent years (Jiuxun, 2005), the universal (EOS) have not been limited to just solid or just liquid, this means that an (EOS) initially proposed for solids might be applied to compressed liquids and vice versa. 
The spinodal is locus in $\mathrm{p}-\mathrm{v}$ diagram of compressed liquids, which is the limit of metastability of substance with respect to a phase transition, the spinodal can be detected in the metastable region, but in practice such experiments are extremely difficult. Instead efforts have been made to locate the spinodal by extrapolation from the stable region of phase diagram. So an (EOS) satisfying the spinodal condition useful to the research on expanded liquids.

Differences shown in table (2) could be interpreted that (jiuxun, 2005) work didn't consider that $\left(\mathrm{P}_{\mathrm{sp}}\right)$ for the material obtained within the metastable state while the extrapolation shown in figure (3) and (4) in the present work evaluate $\left(\mathrm{P}_{\mathrm{sp}}\right)$ within the metastable state of the material.

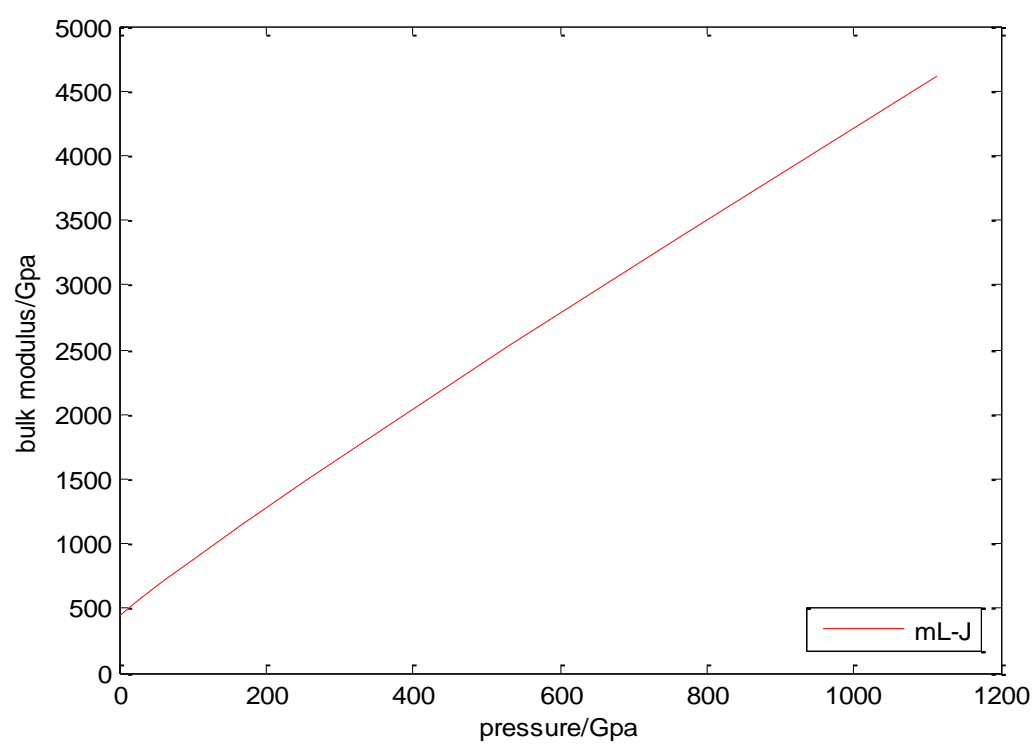

Figure (1): variation of bulk modulus with pressure for carbon

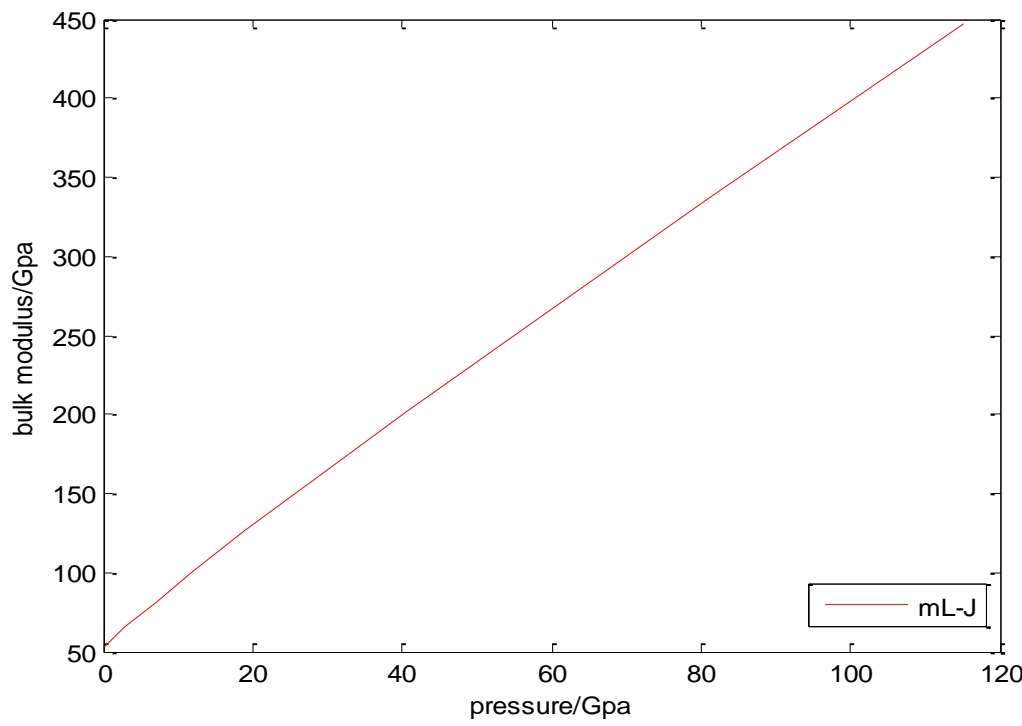

Figure (2): variation of bulk modulus with pressure for $\alpha$-Sn 


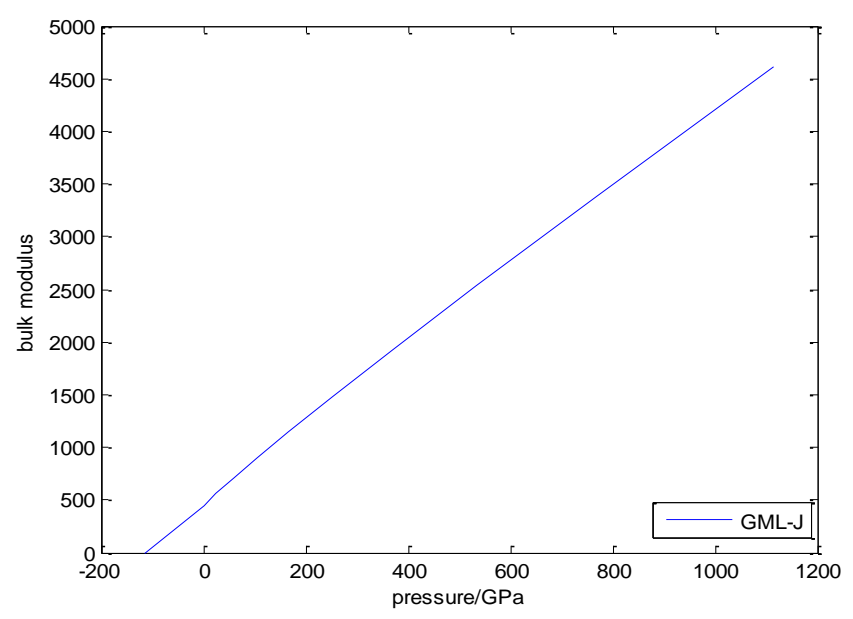

Figure (3): The spinodal pressure for carbon

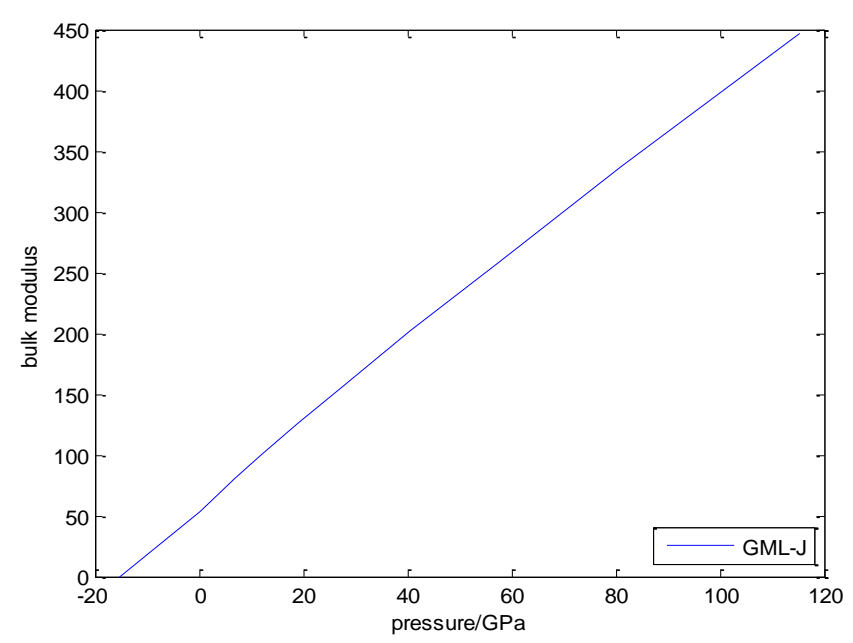

Figure (4): The spinodal pressure for $\alpha-S n$

\section{References:}

1) Gaudion R. and Foulkes, 2002, "Ab initio calculation of bulk modulus and comparisons with experiments", Phys. Rev. B66, Issue $5, \mathrm{P} 052104$.

2) Jiuxun S., 2005, "A modified lennard-jones-type equation of state for solids strictly satisfying the spinodal condition", J. phys.: Condens. Matter 17, L103-L111.

3) Lennard-Jones J.E., 1924 "On the determination of molecular field. II. from the EOS of a Gas" Proc. R. Soc. Lond. A 106,463-477.

4) Maris H.J., 2009, "Properties of metastable Quantum solids", J. Low temp. phys. 158 , No. 3-4, 485-489.

5) Tripathi P., Misra G., Goyal S. C., 2006, "Equation of state for group IV-IV semiconductors", Solid State Communications 139,132-137.

6) Yutaka Tada, Akihiko Tamakoshi, Yoshihito Kato and Yuichiro Nagatsu, 2007, "Generalized peng-Robenson EOS with pair potential parameters for liquid n-Alkanes", fluid phase equilibria 262, Issues 1-2,236-243. 